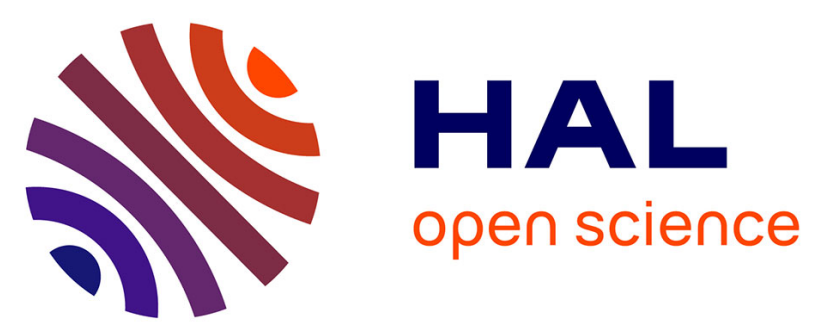

\title{
Evidence that two tilapia (Oreochromis niloticus) prolactins have different osmoregulatory functions during adaptation to a hyperosmotic environment
}

Benoît Aupérin, F. Rentier-Delrue, J.A. Martial, Patrick Prunet

\section{- To cite this version:}

Benoît Aupérin, F. Rentier-Delrue, J.A. Martial, Patrick Prunet. Evidence that two tilapia (Oreochromis niloticus) prolactins have different osmoregulatory functions during adaptation to a hyperosmotic environment. Journal of Molecular Endocrinology, 1994, 12, pp.13-24. 10.1677/jme.0.0120013 . hal-02715347

\author{
HAL Id: hal-02715347 \\ https://hal.inrae.fr/hal-02715347
}

Submitted on 1 Jun 2020

HAL is a multi-disciplinary open access archive for the deposit and dissemination of scientific research documents, whether they are published or not. The documents may come from teaching and research institutions in France or abroad, or from public or private research centers.
L'archive ouverte pluridisciplinaire HAL, est destinée au dépôt et à la diffusion de documents scientifiques de niveau recherche, publiés ou non, émanant des établissements d'enseignement et de recherche français ou étrangers, des laboratoires publics ou privés. 


\section{Evidence that two tilapia (Oreochromis niloticus) prolactins have different osmoregulatory functions during adaptation to a hyperosmotic environment}

\section{B Auperin, F Rentier-Delrue*, J A Martial* and P Prunet}

Laboratoire de Physiologie des Poissons, INRA, Campus de Beaulieu, 35042 Rennes Cédex, France

* Laboratoire de Biologie Moléculaire et de Génie Génétique, Université de Liège, Allée du 6 Août, B6, B-4000 Sart Tilman, Belgium

(Requests for offprints should be addressed to P Prunet)

\begin{abstract}
Two forms of prolactin (tiPRL $\mathrm{I}_{\mathrm{I}}$ and tiPRL $\mathrm{II}_{\mathrm{I}}$ ), with only $69 \%$ sequence identity, have been previously described in the cichlid fish tilapia (Oreochromis species). In the present study we have attempted to investigate the biological activity of these two prolactin forms during adaptation to a hyperosmotic environment. For this purpose, we have developed two highly sensitive (sensitivity: $0.05 \mathrm{ng} / \mathrm{ml}$ ) and specific (cross-reactivity $<0.04 \%$ ) radioimmunoassays for tiPRL $L_{I}$ and tiPRL $L_{I I}$, using recombinant hormones.

When fish were directly transferred from fresh to brackish water, the measured levels of plasma tiPRL $_{I}$ and tiPRL ${ }_{I I}$ dropped abruptly until $12 \mathrm{~h}$ after transfer. Thereafter, plasma tiPRL $\mathrm{II}_{\mathrm{I}}$ remained stable (around $0.5 \mathrm{ng} / \mathrm{ml}$ ) until the end of the experiment, whereas plasma tiPRL $\mathrm{I}_{\mathrm{I}}$ continued to decrease to undetectable levels. These different patterns of change are reflected in the calculated ratio of plasma tiPRL $L_{I I}$ to tiPRL $L_{1}$, which increased from $2-3$ in fresh water-adapted fish to over 10 in fish which had spent 3 days or more in brackish water. The pituitary contents of tiPRL $L_{I}$ and tiPRL $L_{I I}$ varied in a qualitatively similar fashion after transfer to brackish water. The tiPRL $\mathrm{I}_{\mathrm{I}}$ content
\end{abstract}

dropped continuously after $12 \mathrm{~h}$, reaching onetwelfth of its initial level after 2 weeks. The pituitary tiPRL $\mathrm{II}_{1}$ content, on the other hand, did not decrease significantly until day 7 , and after a 2-week exposure to brackish water it had only decreased by $50 \%$.

When injected into tilapia adapted to brackish water, both ovine prolactin and recombinant tiPRL induced a clear dose-dependent ion-retaining effect. In contrast, the effect induced by tiPRL ${ }_{I I}$ treatment was markedly smaller and not dose-dependent.

Northern blot analysis of tiPRL mRNAs using either a tiPRL $L_{I}$ or a tiPRL $\mathrm{II}_{\mathrm{I}} \mathrm{CDNA}$ probe indicated the presence of two mRNAs differing in size: a 1.7 $\mathrm{kb}$ mRNA coding for tiPRL $\mathrm{I}_{\mathrm{I}}$ and a $1.3 \mathrm{~kb}$ mRNA coding for tiPRL $\mathrm{II}_{\mathrm{II}}$. After transfer to brackish water, levels of the two mRNAs decreased similarly.

The present study indicates that, in O. niloticus, the two forms of prolactin have different osmoregulatory roles during adaptation to brackish water. Accordingly, their syntheses are differentially regulated after transfer to a hyperosmotic environment, presumably at a post-transcriptional level.

Fournal of Molecular Endocrinology (1994) 12, 13-24

\section{INTRODUCTION}

Prolactin belongs to a family of structurally and functionally related polypeptides which also includes growth hormone $(\mathrm{GH})$, placental lactogen, proliferin and somatolactin. Although in man, rats and turkeys prolactin is the product of a single gene, the hormone occurs in multiple molecular forms (cleaved, phosphorylated or glycosylated; Lewis et al. 1984; Clapp et al. 1989; Brooks et al. 1990), whose discrete functions remain a matter of debate.
In teleost fish, nucleotide and polypeptide sequence analyses have revealed the existence of two distinct, albeit similar, genes in some species. Such is the case for salmon, Oncorhynchus keta, and for carp, Cyprinus carpio (Yasuda et al. 1986, 1987). In tilapia, two much less similar prolactin molecules have been characterized; their sequence identity is only $69 \%$, one variant (tilapia prolactin $\mathrm{I}_{\text {, tiPR }} \mathrm{L}_{\mathrm{I}}$ ) being more similar to other fish prolactins than to the other variant $\left(\mathrm{tiPRL}_{\mathrm{II}}\right.$ ) (Yamaguchi et al. 1988; Rentier-Delrue et al. 1989). 
In fish, prolactin exhibits a pleiotropic spectrum of biological activities including effects on osmoregulation, metabolism and behaviour (for a review see Clarke \& Bern, 1980). However, the primary and most studied role of prolactin in fish is water and electrolyte homeostasis (Loretz \& Bern, 1982; Hirano, 1986; Prunet et al. 1990). In fresh water, treatment of hypophysectomized tilapia (Oreochromis mossambicus) with mammalian or fish prolactin restores the osmolarity and electrolyte concentrations to normal levels (Dharmamba et al. 1967; Dharmamba, 1970; Specker et al. 1985), thus demonstrating that prolactin is necessary for survival in a hypo-osmotic environment. Furthermore, when injected into sea water-adapted tilapia, ovine prolactin profoundly disturbs the hydromineral balance by inhibiting salt extrusion by gill chloride cells (Dharmamba \& Maetz, 1972; Dharmamba et al. 1975; Foskett et al. 1983).

Both tiPRLs behave similarly in restoring the hydromineral balance of hypophysectomized tilapia transferred to fresh water (Specker et al. 1985; Young et al. 1988). However, Specker et al. (1989) have shown that the salamandrid integumental bioassay can distinguish between the two prolactin variants.

To analyse possible differences in biological function between the two variants further, we have produced recombinant tiPRLs and used multiple approaches to study their osmoregulatory roles during adaptation to a hyperosmotic environment. With radioimmunoassays (RIAs) specific for each form of prolactin, we have measured plasma and pituitary levels after direct transfer from fresh water to brackish water. We additionally analysed both tiPRL $_{I}$ and tiPRL ${ }_{I I}$ mRNAs. We also report the effects of injected tiPRL $L_{I}$ and tiPRL ${ }_{I I}$ on electrolyte homeostasis in tilapia adapted to brackish water. Our results clearly demonstrate that the two prolactins perform different biological functions during adaptation to a hyperosmotic environment, and that they are differentially regulated.

\section{MATERIALS AND METHODS}

\section{Sources of hormones and antisera}

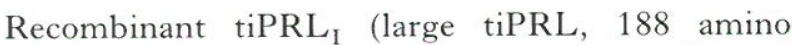

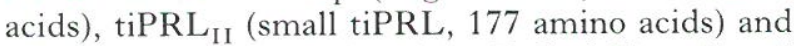
tilapia GH were produced in Escherichia coli and further purified (Rentier-Delrue et al. 1989; Swennen et al. 1991). Ovine prolactin (NIDDKoPRL-19) was supplied by the National Institute of Arthritis and Metabolic Diseases, NIH, Bethesda, MD, U.S.A. Chinook salmon prolactin was purified according to Prunet \& Houdebine (1984). Antisera against recombinant tiPRL $L_{I}$ and tiPRL $L_{I I}$ were raised in rabbits.

\section{Iodination of tiPRL}

The two tiPRLs were iodinated according to the chloramine-T method (Greenwood et al. 1963) modified by Martal (1972). Specific activities were estimated (about $40-50 \mu \mathrm{Ci} / \mu \mathrm{g}$ for tiPRL $\mathrm{I}_{\mathrm{I}}$ and $50-60 \mu \mathrm{Ci} / \mu \mathrm{g}$ for tiPRL $\mathrm{II}_{\mathrm{II}}$ ) from the rate of iodine incorporation into prolactin. Labelled hormone was stored in $50 \%$ glycerol at $-20{ }^{\circ} \mathrm{C}$.

\section{Radioimmunoassay procedure}

The same procedure was used for both tiPRL $\mathrm{I}_{\mathrm{I}}$ and tiPRL $L_{I I}$. RIAs were performed using a double antibody method under non-equilibrium conditions as described by Prunet et al. (1985), with minor differences. The second precipitation was obtained by adding sheep anti-rabbit gamma globulin antiserum to each tube without polyethylene glycol.

In order to validate these RIAs, plasma and pituitaries from different tilapia species (O. niloticus, $O$. aureus and Sarotherodon melanotheron) were collected on the Ivory Coast from adult fish. Morever, plasma from hypophysectomized adult (250-300 g) O. niloticus specimens was also tested. Hypophysectomy was performed as described by Nishioka (1980) and blood was collected 24 and $48 \mathrm{~h}$ after surgery.

\section{Transfer experiment}

Tilapia specimens (O. niloticus) supplied by IDESSA (Bouaké, Ivory Coast) were reared in recirculating fresh water at a constant temperature $\left(26{ }^{\circ} \mathrm{C}\right)$ and with a constant photoperiod $(12 \mathrm{~h}$ light:12 h darkness) for 7 months.

Two similar experiments were performed, one over a 14-day period (with fish having a weight of $91 \pm 1 \mathrm{~g}($ mean \pm S.E.M., $n=8))$ and another over a 28-day period (long-term adaptation, with fish having a weight of $134 \pm 3 \mathrm{~g}$ ). Fish were transferred to aquaria (eight fish per 80 litre aquarium with filter) and kept in fresh water for 2 weeks before starting the experiment. Transfer to brackish water $(20 \%$ salinity) was effected by adding an adequate amount of concentrated salt water made with marine salt. Thus, transfer to the hyperosmotic environment was nearly instantaneous. The water temperature during the experiment was the same in the fresh water and brackish water aquaria $\left(26-27^{\circ} \mathrm{C}\right)$ and the photoperiod was $12 \mathrm{~h}$ light: $12 \mathrm{~h}$ darkness in both cases. Neither group was starved after transfer. Two-thirds of the water was changed twice a week. 
Eight fish from a fresh water aquarium and eight from a brackish water aquarium were sampled at each point in the experiment. On the day of transfer, a sample of seven fish, representative of the whole fresh water population, was taken as a control group.

\section{Plasma and pituitary collection}

To avoid the effect of stress on plasma prolactin levels, the fish were caught individually by hand-net in each aquarium. This technique proved to be preferable to anaesthesia, as the latter significantly increases the plasma levels of tiPRL $\mathrm{I}_{\mathrm{I}}$ and tiPRL $\mathrm{L}_{I I}$ (B. Auperin \& P. Prunet, unpublished results). Within $1 \mathrm{~min}$ of catching each fish, and before collecting the pituitary, blood was collected by caudal puncture with a heparinized syringe. After blood centrifugation, plasma was collected and frozen at $-20{ }^{\circ} \mathrm{C}$. Pituitaries were immediately frozen in liquid nitrogen and stored at $-20{ }^{\circ} \mathrm{C}$ until assayed.

\section{Hormonal treatment of $O$. niloticus adapted to brackish water}

Tilapia (O. niloticus), with a mean weight of $20 \pm 0.3 \mathrm{~g}$ and adapted to an aquarium, were directly transferred to brackish water $\left(20^{\circ} \%\right.$ salinity) at a constant temperature of $27^{\circ} \mathrm{C}$, and maintained at this salinity for 2 weeks prior to the start of the experiment. No mortality was observed during the period of adaptation and fish were fed at a rate of $1 \%$ body weight per day before and during the experiment. Fish were further distributed into four aquaria (capacity 80 litres; 9 or 10 fish per aquarium) and injected intraperitoneally every 2 days for 8 days. On the day after the last injection, blood was collected, centrifuged and frozen at $-20{ }^{\circ} \mathrm{C}$ until analysis.

Each group of fish received one of the following injections: saline $(\mathrm{NaCl}, 0.15 \mathrm{M}$ in $1 \mathrm{ml}$ for $50 \mathrm{~g}$ fish); recombinant tiPRL $10 \cdot 1,0.25$ or $0.5 \mu \mathrm{g} / \mathrm{g}$ body weight, dissolved in $10 \mathrm{~mm} \mathrm{NH}_{4} \mathrm{HCO}_{3}$ $(1 \mu \mathrm{g} / 2 \mu \mathrm{l})$ and diluted with $0 \cdot 15 \mathrm{M} \mathrm{NaCl}$ to the desired concentration); recombinant tiPRL $\mathrm{II}_{\mathrm{I}}$ (same concentrations as for tiPRL $\left.\mathrm{I}_{\mathrm{I}}\right)$; ovine prolactin $(4,10$ or $20 \mu \mathrm{g} / \mathrm{g}$ body weight, prepared in the same manner as tiPRL). In order to test the different doses mentioned above, three independent experiments were performed at the same time with fish from the same stock.

\section{Ion measurements}

The chloride level was measured by amperometric titration (Isa Biologie CL 500; Isa Biologie,
Morangis, France) of $20 \mu \mathrm{l}$ undiluted plasma. The plasma sodium level was determined by absorption spectrophotometry using plasma samples diluted 4000 times.

\section{RNA analysis}

Pituitaries from tilapia (O. niloticus) reared in fresh water or adapted over 2 weeks to brackish water $(20 \%$ salinity) were collected and immediately frozen in liquid nitrogen.

Total RNA was prepared according to the method of Chomczynski \& Sacchi (1987). Tilapia mRNA was further size-fractionated on formaldehyde-agarose gels, and then transferred to nylon membranes (Biodyn-Pall; Pall Europe Ltd, Portsmouth, Hants, U.K.). All hybridization was performed under conditions of high stringency ('Thomas, 1980). The probes used in these hybridization experiments were the full-length (coding and non-coding sequences) tiPRL $\mathrm{I}_{\mathrm{I}}$ and tiPRL $_{\text {II }}$ cDNAs inserted into pUC13 and cut at the EcoRI sites as described by Rentier-Delrue et al. (1989). We kept both coding and non-coding sequences in order to have most dissimilarity between the two tiPRLs. To control for variation in sample loading, the nylon membranes were further stripped and reprobed for $\beta$-actin mRNA using rainbow trout cDNA (Pakdel et al. 1989). All cDNAs were labelled with a random-priming DNA labelling kit and $\left[\alpha-{ }^{32} \mathrm{P}\right] \mathrm{dCTP}(800 \mathrm{Ci} / \mathrm{mmol}$; Amersham, Les Ulis, France). The band intensities were quantified by densitometric scanning of autoradiograms. The two mRNA species $(1.3$ and $1.7 \mathrm{~kb})$ revealed on blots hybridized with either the tiPRL $_{I}$ cDNA or the tiPRL II $c$ DNA probe were scanned, and quantification of these bands allowed us to calculate the ratio of tiPRL $\mathrm{II}_{\text {mRNA to }}$ tiPRL $_{\mathrm{I}}$ mRNA for different pituitary samples. This quantification was performed with samples showing different quantities of tiPRL mRNAs and with different amounts of total RNA (3 or $5 \mu \mathrm{g}$ ) loaded on the gel. Autoradiograms were exposed for several periods and scanning analysis was performed on films where signals were not saturated.

\section{Statistical analysis}

The Mann-Whitney test was used to characterize differences between the means from experiments involving treatment or transfer, after variance analysis with nested factors (analysis of variance, with transfer or treatments and time as factors). Results are presented as means \pm S.E.M. Significance was accepted at the 5\% level. 

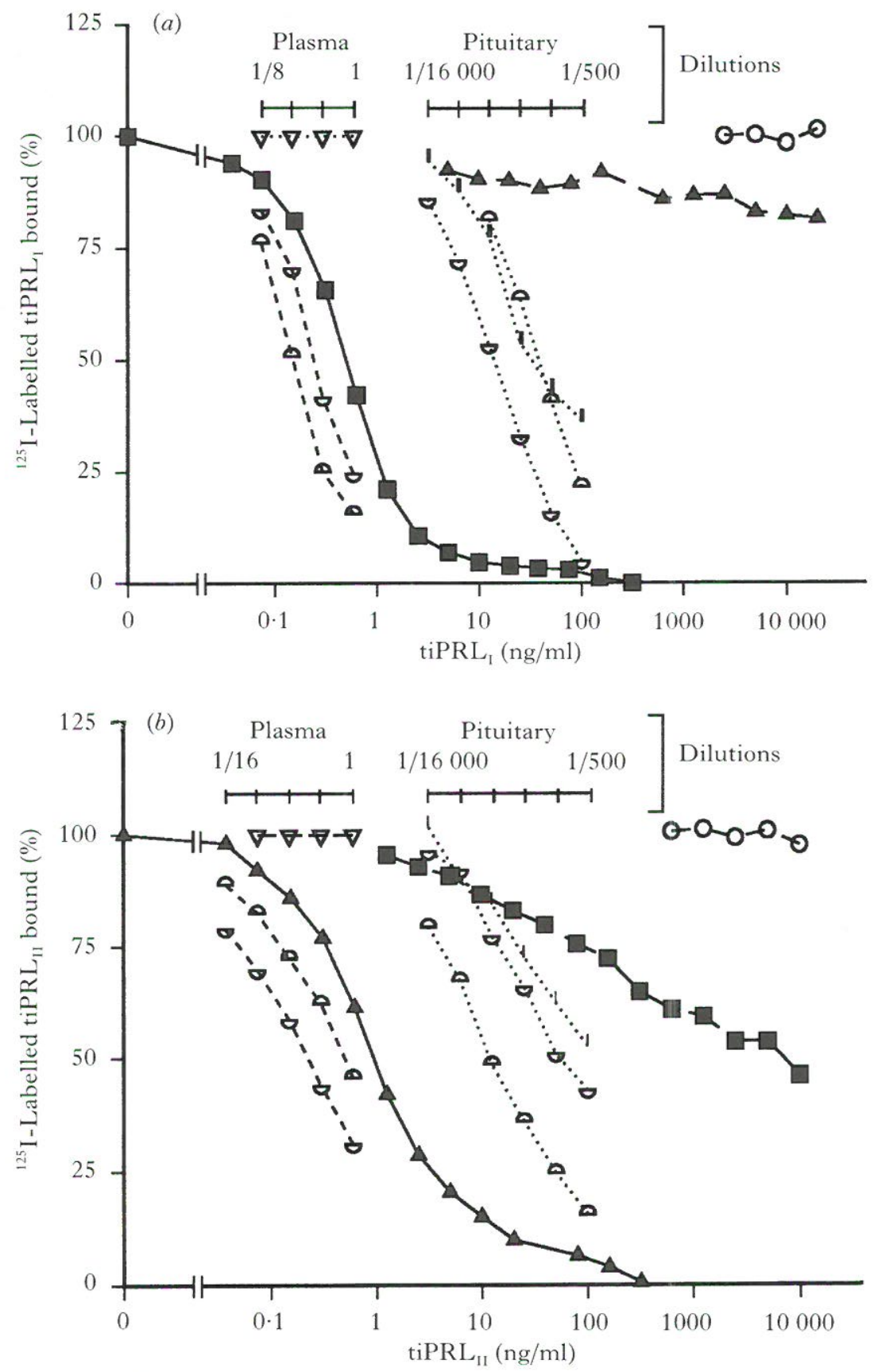

FIGURE 1. Radioimmunoassays for recombinant (a) tilapia prolactin I $\left(\right.$ tiPRL $\left._{\mathrm{I}}\right)$ and $(b)$ tiPRL $_{\mathrm{II}}$, showing the displacement of ${ }^{125} \mathrm{I}$-labelled tiPRL or ${ }^{125} \mathrm{I}_{\text {-labelled tiPRL }}$ by the tiPRL $\mathrm{I}_{\mathrm{I}}(\boldsymbol{\square})$ and tiPRL $\mathrm{LII}_{\mathrm{I}}(\boldsymbol{\Delta})$ standards, different hormonal preparations (tilapia GH, ovine PRL, salmon PRL; O), pituitary homogenate (dotted lines) and plasma (dashed lines) sample dilutions (as shown) from different fresh water-reared Cichlid species (Oreochromis niloticus $(\triangle)$, O. aureus $(\square)$ and Sarotherodon melanotheron $(\mathbf{I})$ ) and plasma from hypophysectomized $O$. niloticus $(\nabla)$. Each point is the mean of duplicate determinations. ${ }^{125} \mathrm{I}$-Labelled PRL bound (\%) refers to

${ }^{125}$ I-labelled PRL bound as a percentage of the label bound in the absence of tiPRL. 


\section{RESULTS}

\section{Validation of the RIAs for tiPRL $\mathrm{I}_{\mathrm{I}}$ and tiPRL} (Fig. 1)

When tested in the corresponding RIAs, serial dilutions of pituitary extracts and plasma from $O$. niloticus, $O$. aureus and $S$. melanotheron produced


inhibition curves that paralleled the standard curves for tiPRL $\mathrm{I}_{1}$ or tiPRL $\mathrm{II}_{\mathrm{I}}$. Plasma from hypophysectomized $O$. niloticus failed to displace labelled hormone in both RIAs. Assay specificity was further confirmed by the absence of cross-reactivity with tilapia $\mathrm{GH}$, ovine prolactin and salmon prolactin. Furthermore, tiPRL ${ }_{I I}$ did not cross-react in the tiPRL $L_{I}$ RIA, nor did tiPRL $L_{1}$ exhibit significant cross-reactivity in the tiPRL ${ }_{I I}$ RIA $(0 \cdot 04 \%$ for a $50 \%$ displacement $)$.

The mid-range value, calculated as the amount of tiPRL required to bind $50 \%$ of the zero-hormone standard $\left(\mathrm{ED}_{50}\right)$ was $0.493 \pm 0.013 \mathrm{ng} / \mathrm{ml}$ for tiPRL and $0.938 \pm 0.029 \mathrm{ng} / \mathrm{ml}$ for tiPRL $L_{I I}$. The estimated assay sensitivity, defined as twice the S.D. at zero dose, was $0.05 \mathrm{ng} / \mathrm{ml}$ in both assays. These values tally well with the plasma levels of both prolactins.

The effects of plasma components on the assay were examined by determining the recovery of tiPRL $_{I}$ or tiPRL ${ }_{I I}$ in the presence of $50 \mu$ plasma from hypophysectomized $O$. niloticus. The slope of the regression line between prolactin added and recovered did not differ significantly from 1 when the amounts used did not exceed $4 \mathrm{ng} / \mathrm{ml}$ in the tiPRL, RIA or $5 \mathrm{ng} / \mathrm{ml}$ in the tiPRL ${ }_{I I}$ assay. Above these concentrations, the prolactin concentration was slightly underestimated (data not shown). Repeated determinations of a pool of $O$. niloticus plasma gave intra- and interassay coefficients of variation of $8 \cdot 0 \%(n=10)$ and $10.5 \%(n=4)$ for tiPRL 1 and $7 \cdot 3 \%(n=10)$ and $12 \cdot 2 \%(n=4)$ for tiPRL $_{I I}$ respectively.

\section{Changes in plasma and pituitary prolactin levels during adaptation to brackish water (Figs 2 and 3)}

Within $3 \mathrm{~h}$ of direct transfer to brackish water, plasma $\mathrm{Cl}^{-}$levels were found to have increased significantly (data not shown). The highest levels were observed on the day after transfer (day 1) $(200 \pm 3 \cdot 7$ versus $138 \pm 1.5 \mathrm{mEq} / \mathrm{l}$ in fresh water

FIGURE 2. Changes in plasma $(a)$ tilapia prolactin I $\left(\right.$ tiPRL $\left._{\mathrm{I}}\right)$ and $(b)$ tiPRL $_{\mathrm{II}}$ levels and $(c)$ the plasma tiPRL $_{I I}:$ tiPRL $_{I}$ ratio of Oreochromis niloticus after direct transfer from fresh water to $20 \%$ brackish water (triangles) or retention in fresh water (squares). Values shown are means \pm S.E.M. $(n=8$ or 9$)$. ${ }^{*} P<0 \cdot 05$, ** $P<0.01$ compared with values for fresh water fish (the Mann-Whitney test was used to characterize differences between means after variance analysis with nested factors, with transfer and time as factors). ND: no detectable values. 
fish, $n=8$ ). From then on, however, the $\mathrm{Cl}^{-}$levels dropped, stabilizing at $10 \%$ above the fresh water values on day 3 . This shows that the fish were able to regulate their hydromineral balance. Plasma $\mathrm{Na}^{+}$ levels exhibited the same pattern of change (data not shown).
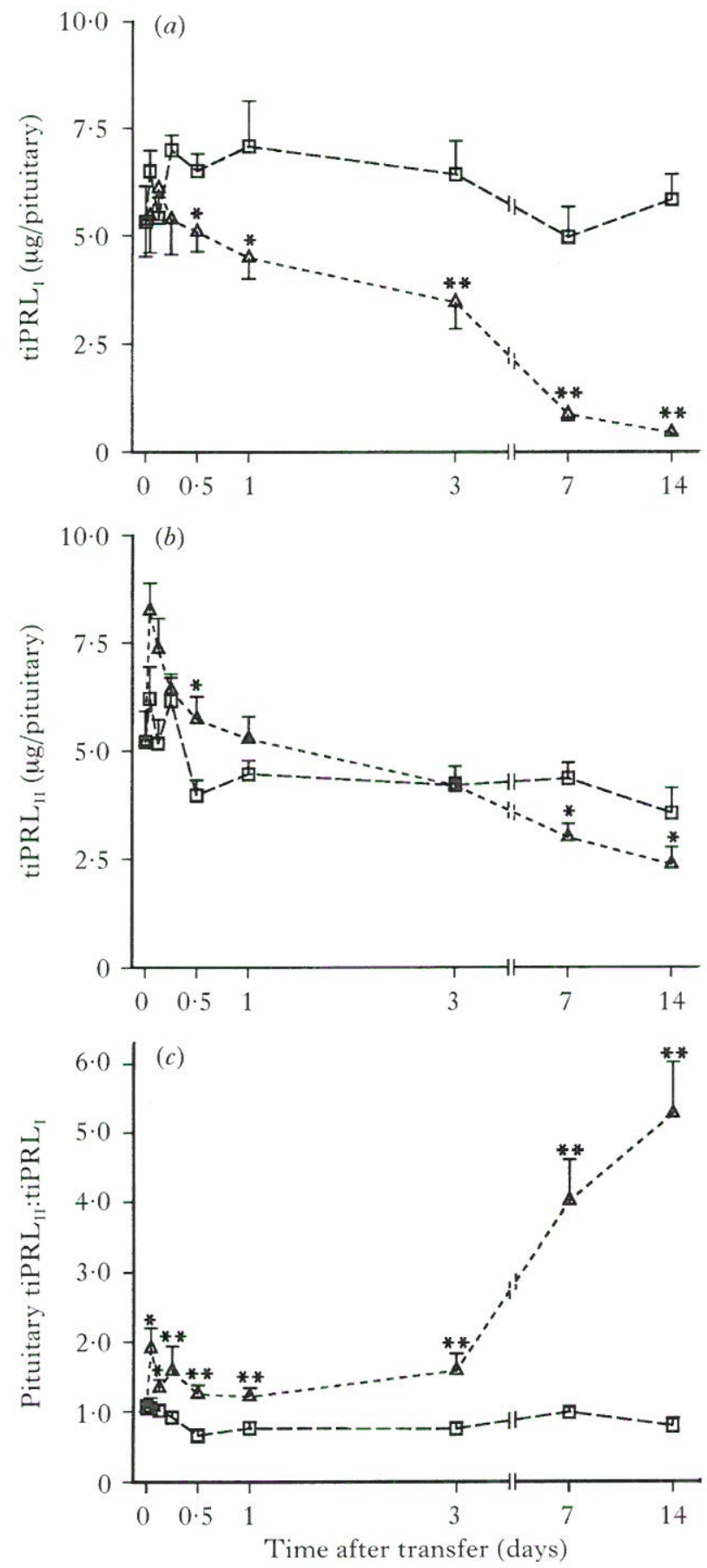

As early as $6 \mathrm{~h}$ after exposure to brackish water, the levels of both plasma prolactins dropped abruptly. This trend continued until $12 \mathrm{~h}$ after transfer, after which the tiPRL $\mathrm{L}_{\mathrm{II}}$ level remained low $(0.5 \mathrm{ng} / \mathrm{ml})$ until the end of the experiment (on day 14), whereas the tiPRL $\mathrm{I}_{\mathrm{I}}$ level continued to drop. By day 7, tiPRL $_{\mathrm{I}}$ was undetectable and remained so on day 14. In the long-term adaptation experiment, the hormone levels on day $28(0.13 \pm 0.01 \mathrm{ng} / \mathrm{ml}$ for tiPRL $\mathrm{I}_{\mathrm{I}}$ and $1.058 \pm 0 \cdot 155 \mathrm{ng} / \mathrm{ml}$ for tiPRL $\left.\mathrm{II}_{\mathrm{I}}\right)$ did not differ significantly from those measured on day $14(0.12 \pm 0.002$ and $0.66 \pm 0.107 \mathrm{ng} / \mathrm{ml}$ respectively).

The calculated ratio of plasma tiPRL $\mathrm{II}_{\text {II }}$ to plasma tiPRL $_{I}$ clearly reflects these differences in the way the two levels change after exposure to brackish water. From a value of between 2 and 3 in fresh water, this ratio rose to over 10 after a 3 -day exposure.

In the transfer experiment, fish weights remained fairly homogenous (less than $8 \%$ variability), so prolactin contents per pituitary could be directly compared in the different groups. Twelve hours after transfer, a significant drop in pituitary tiPRL was observed. Thereafter, the tiPRL $L_{I}$ content continued to decrease, and after a 2-week exposure to brackish water it was 12 times lower than the values observed in fresh water controls. Changes in tiPRL ${ }_{I I}$ content after transfer followed a different pattern; the tiPRL $\mathrm{II}_{\text {I }}$ content did not drop significantly until 7 days after transfer. By the end of the experiment, the tiPRL ${ }_{\mathrm{II}}$ content had decreased by only half when compared with the control group. In long-term-adapted fish (28 days) the prolactin contents $\left(0.465 \pm 0.02 \mu \mathrm{g} /\right.$ pituitary for tiPRL $\mathrm{I}_{\mathrm{I}}$ and $3.762 \pm 0.627 \mu \mathrm{g} /$ pituitary for tiPRL $\mathrm{II})$ did not differ significantly from those measured in the same experiment on day $14(0.586 \pm 0.102$ and $4 \cdot 057 \pm 1 \cdot 040 \mu \mathrm{g} /$ pituitary respectively).

Here again, the tiPRL $L_{I I}:$ tiPRL $_{I}$ ratio reflects how differently the amounts of tiPRL $L_{I}$ and tiPRL ${ }_{I I}$ in the pituitaries changed in response to transfer. This ratio increased from 1 before transfer to 5 after adaptation to brackish water.

FIGURE 3. Changes in pituitary $(a)$ tilapia prolactin I $\left(\right.$ tiPRL $\left._{\mathrm{I}}\right)$ and $(b)$ tiPRL $_{\mathrm{II}}$ levels and $(c)$ the pituitary tiPRL ${ }_{I I}:$ tiPRL $_{I}$ ratio of Oreochromis niloticus after direct transfer from fresh water to $20 \%$ brackish water (triangles) or retention in fresh water (squares). Values shown are means \pm S.E.M. $(n=8$ or 9$)$. ${ }^{*} P<0 \cdot 05$, ** $P<0.01$ compared with values for fresh water fish (the Mann-Whitney test was used to characterize differences between means after variance analysis with nested factors, with transfer and time as factors). 


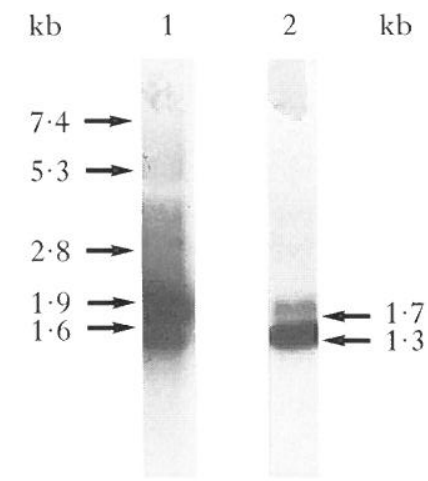

FIGURE 4. Northern blot analysis of mRNA extracted from 20 fresh water-adapted tilapia pituitaries. Total RNA $(5 \mu \mathrm{g})$ was denatured, electrophoresed and transferred to nylon membranes. The filter was successively hybridized with tilapia prolactin I ( tiPRL $\left._{I}\right)$ cDNA (panel 1) and tiPRL ${ }_{1 I}$ cDNA (panel 2) (exposure times: tiPRL, 2 days; tiPRL ${ }_{I I}, 4$ days). A control autoradiograph was made after each dehybridization. The sizes of RNA molecular markers $(\mathrm{kb})$ are indicated on the left.

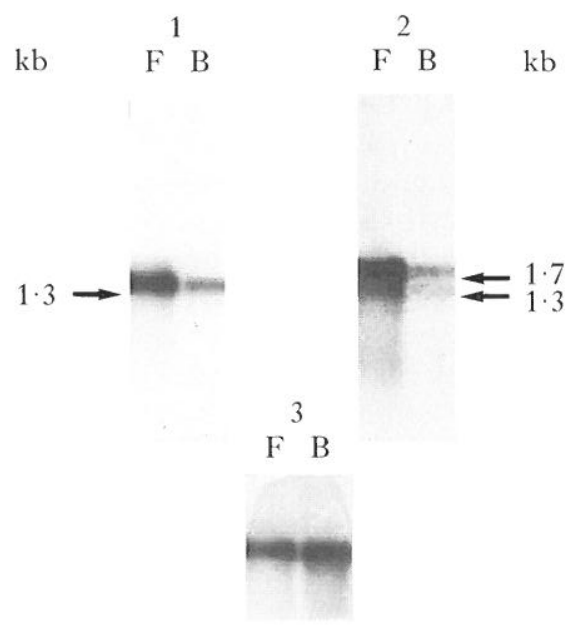

FIGURE 5. Northern blot analysis of mRNA extracted from 20 pituitaries collected from fresh water-adapted (F) and brackish water-adapted (B) tilapia (Oreochromis niloticus). Total RNA (5 $\mu$ g) was denatured, electrophoresed and transferred to nylon membranes. The filter was successively hybridized for tilapia prolactin II (tiPRL $\left.{ }_{I I}\right)$ (panel 1), tiPRL (panel 2) and actin (panel 3) (exposure times: tiPRL $\mathrm{II}_{\mathrm{I}}, 6$ days; tiPRL $\mathrm{t}_{1}$, 7 days; actin, 8 days). A control autoradiograph was made after each dehybridization.

\section{Analysis of tiPRL mRNA (Figs 4, 5 and 6)}

Tilapia prolactin mRNAs were analysed by Northern blotting, and the same membranes were successively hybridized with tiPRL $\mathrm{II}_{\text {I }}$ and tiPRL

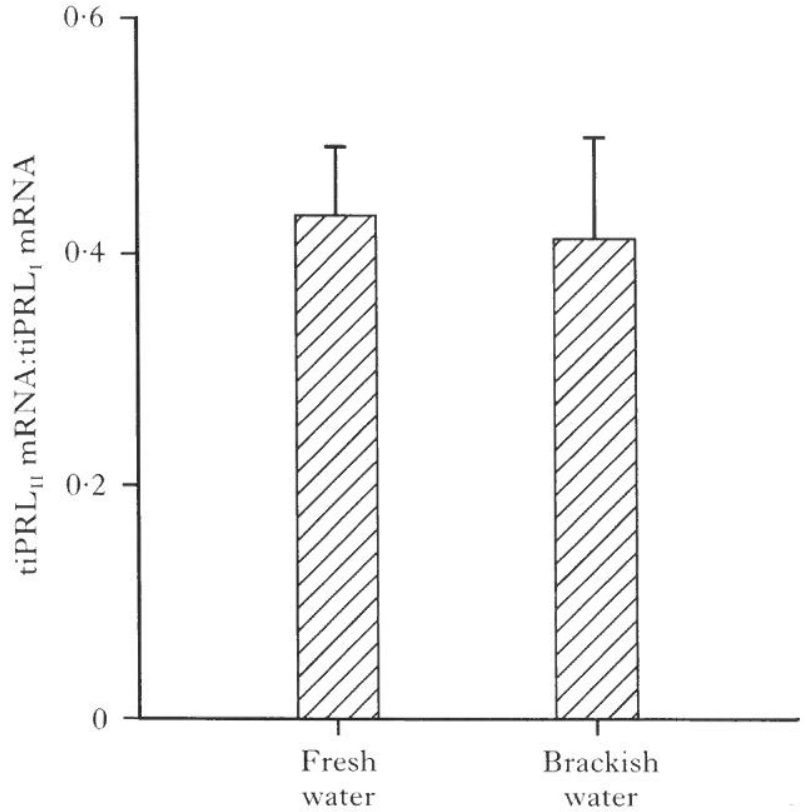

FIGURE 6. Ratio of tilapia prolactin II $\left(t_{i P R L}\right)$ mRNA to tiPRL $\mathrm{I}_{\mathrm{I}} \mathrm{mRN}$ in tilapia adapted to fresh water or transferred for 2 weeks to brackish water. Total RNA was extracted from ten pituitaries collected from fish adapted to fresh or brackish water. Pituitary PRL mRNAs were measured after Northern blot analysis

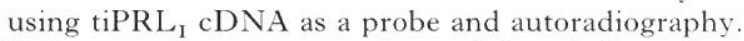
Values are given as means $\pm S$.E. of three independent Northern blots corresponding to three independent transfer experiments. The ratio in brackish water was not significantly different from that in fresh water (Mann-Whitney non-parametric test).

cDNA probes. Using conditions of high stringency, two bands of different sizes $(1.7$ and $1.3 \mathrm{~kb})$ and intensities were observed with each probe (Fig. 4). Interestingly, whereas for tiPRL $\mathrm{I}_{\mathrm{I}} \mathrm{CDNA}$ the more intense signal corresponded to the $1.7 \mathrm{~kb}$ mRNA, the tiPRL $\mathrm{II}_{\mathrm{I}}$ probe gave the strongest signal with the $1.3 \mathrm{~kb}$ mRNA. As hybridization was performed under conditions of high stringency, we conclude that the $1.7 \mathrm{~kb}$ mRNA was tiPRL $\mathrm{I}_{\mathrm{I}}$ mRNA, whereas the $1.3 \mathrm{~kb}$ species was the tiPRL II mRNA. However, these results also indicate that the tiPRL

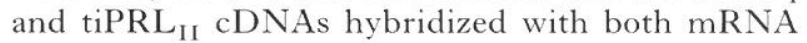
species. When comparing fish adapted to fresh water and those adapted to brackish water, a large decrease in both tiPRL ${ }_{1}$ and tiPRL ${ }_{I I}$ mRNA was observed (Fig. 5). We were further interested to compare the ratio of tiPRL II $m R N A$ to tiPRL mRNA in tilapia adapted to fresh water and brackish water. Calculated from Northern blots hybridized with tiPRL $\mathrm{I}_{\mathrm{I}} \mathrm{CDNA}$, this ratio did not change significantly after exposure to brackish water, thus indicating that both mRNAs decreased 

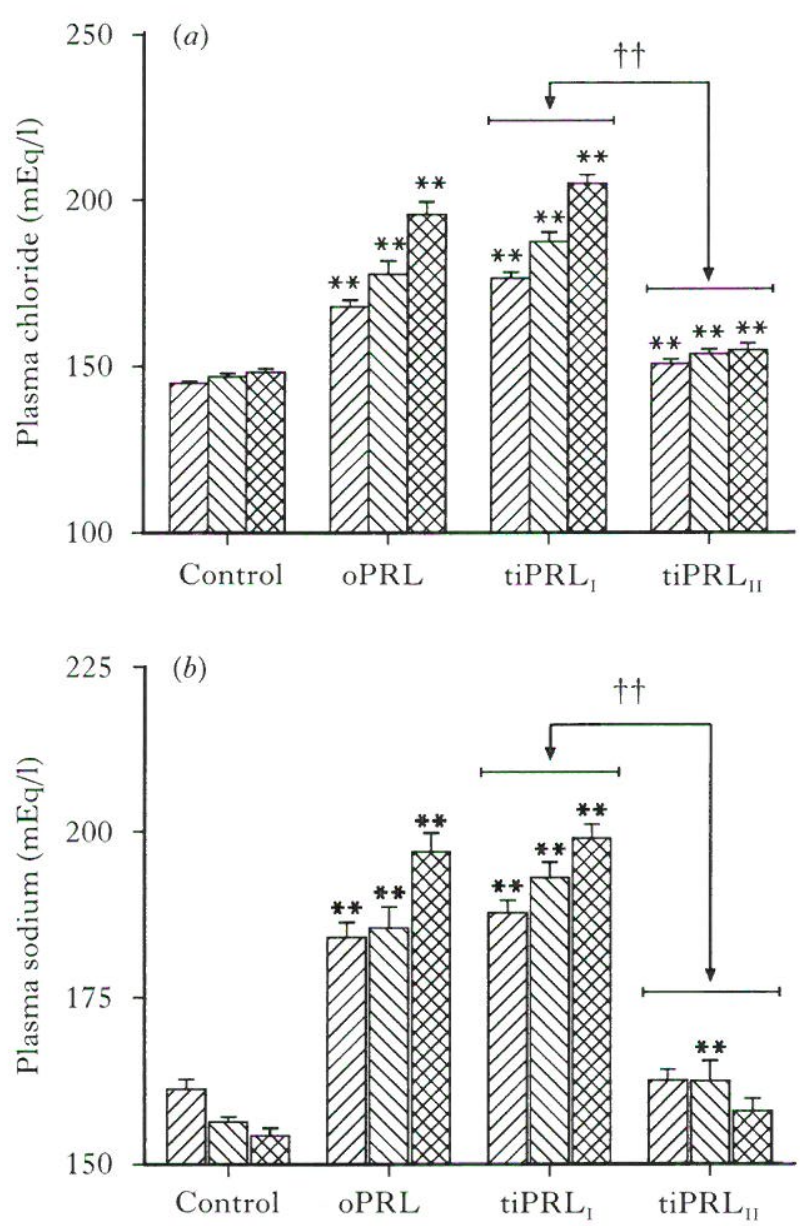

FIGURE 7. Effect of different doses of recombinant tilapia prolactin I $\left(\right.$ tiPRL $\left._{\mathrm{I}}\right)$, recombinant tiPRL $\mathrm{II}_{\mathrm{I}}$ and ovine (o)PRL on plasma $(a)$ chloride and $(b)$ sodium values. Values are shown as means \pm S.E.M. $(n=8$ or 9$)$. ** $P<0.01$ compared with control; $\uparrow+P<0 \cdot 01$, tiPRL $_{\text {II }}$ treatment vs corresponding treatment with tiPRL $\mathrm{I}_{\mathrm{I}}$ (Mann-Whitney non-parametric test). For each treatment, bar 1 represents the low dose (oPRL, $4 \mu \mathrm{g} / \mathrm{g}$; tiPRL $_{I}$ or tiPRL $\left.{ }_{I I}, 0 \cdot 1 \mu \mathrm{g} / \mathrm{g}\right)$, bar 2 represents the medium dose (oPRL, $10 \mu \mathrm{g} / \mathrm{g}$; tiPRL or tiPRL $\mathrm{tI}_{\mathrm{II}}$, $0 \cdot 25 \mu \mathrm{g} / \mathrm{g})$ and bar 3 represents the high dose (oPRL, $20 \mu \mathrm{g} / \mathrm{g}$; tiPRL or tiPRL $_{\mathrm{II}}, 0.5 \mu \mathrm{g} / \mathrm{g}$ ). mEq: milliEquivalent.

similarly after transfer (Fig. 6). A similar result was observed when using blots hybridized with tiPRL cDNA (data not shown).

\section{Effect of prolactin treatment in $O$. niloticus adapted to brackish water (Fig. 7)}

When injected into tilapia specimens adapted to brackish water, both ovine prolactin and recombinant tiPRL ${ }_{I}$ induced a clearly dose- dependent ion-retaining effect, as indicated by the increased plasma $\mathrm{Na}^{+}$and $\mathrm{Cl}^{-}$levels. Furthermore, tiPRL $_{\mathrm{I}}$ was between 50 and 100 times more potent than ovine prolactin. In contrast, tiPRL $\mathrm{II}_{\text {I }}$ only slightly increased the plasma $\mathrm{Na}^{+}$and $\mathrm{Cl}^{-}$levels. The effect was not dose-dependent and was significantly smaller than the effect of either ovine prolactin or tiPRL $L_{I}$.

\section{DISCUSSION}

Since the first description of two prolactins displaying only $69 \%$ similarity in their amino acid sequences in the fish species Oreochromis (Specker et al. 1985; Yamaguchi et al. 1988; Rentier-Delrue et al. 1989), several unsuccessful attempts have been made to distinguish the two prolactin molecules on a functional basis.

The availability of large amounts of active recombinant tiPRLs showing full biological activity (Swennen et al. 1991) has enabled us to investigate differences in the control of osmoregulation by the two prolactins during adaptation to a hyperosmotic environment. To this end, we have developed a highly specific RIA for each prolactin. The two prolactins being quite similar $(69 \%)$, it was necessary to control the specificity of each RIA carefully. No significant cross-reactivity $(<0 \cdot 04 \%)$ was observed between the antisera raised against tiPRL $_{I}$ and tiPRL $\mathrm{II}_{\mathrm{II}}$. Furthermore, no crossreaction was observed with either tilapia $\mathrm{GH}$ or plasma from hypophysectomized fish, whereas the displacement curves of plasma and pituitary extracts from various cichlid species were parallel to the standard curves in both assays. Importantly, both RIAs are very sensitive, as shown by their $E_{50}$ values and the lowest detectable concentrations. They appear to be at least five times more sensitive than previously reported RIAs for fish prolactins or GHs (Nicoll et al. 1981; Hirano et al. 1985; Prunet et al. 1985; Le Bail et al. 1991; Suzuki \& Hirano, 1991; Ayson et al. 1993). This confirms that our RIAs, developed with antisera raised against recombinant tiPRL $L_{I}$ and tiPRL $_{I I}$, are perfectly suitable for measuring plasma and pituitary levels of both tiPRLs.

These RIAs enabled us to investigate the osmoregulatory functions of both prolactins during the adaptation of $O$. niloticus to a hyperosmotic environment. Our first approach was to determine plasma and pituitary levels of both forms at various times prior to and after transfer from fresh to brackish water. As described for several fish species (Nicoll et al. 1981; Hirano et al. 1985; Prunet et al. 1985; Suzuki \& Hirano, 1991; Ayson et al. 1993), 
plasma prolactin levels are higher in fish kept in fresh water than in ones adapted to a hyperosmotic environment. Our results, however, highlight interesting differences between the two forms. Although both prolactin levels rapidly decreased upon transfer to brackish water, their patterns of change differed; the plasma tiPRL, level continued to drop after the first day, reaching undetectable or barely detectable levels by the seventh day, while the plasma tiPRL $\mathrm{II}_{\mathrm{II}}$ level, after an initial drop during the first half-day, then stabilized near $0.5 \mathrm{ng} / \mathrm{ml}$ and remained practically constant, even after 28 days in brackish water. The following features emerge from our results. Under the conditions used (1) the plasma level of tiPRL $L_{I I}$ was always higher than that of tiPRL ${ }_{I}$, (2) the plasma tiPRL ${ }_{I I}:$ tiPRL $_{I}$ ratio increased markedly after transfer, (3) in fish adapted to brackish water, tiPRL $L_{I}$ was no longer detectable in the plasma whereas a significant level of tiPRL $\mathrm{II}_{\mathrm{II}}$ could still be measured. We stress that such differences can only be observed with very sensitive and specific assays, such as the two RIAs developed in the present study. Ayson et al. (1993) reported a low but significant plasma level of tiPRL $\mathrm{I}_{\mathrm{I}}$ in sea water-adapted tilapia $(O$. niloticus $)$ and, in their experiments, they observed a decreased ratio of plasma tiPRL $L_{I I}$ to tiPRL $L_{I}$ after transfer to brackish water. Beyond possible problems of assay sensitivity, these data might have been hampered by sampling stress; 2-phenoxyethanol, used by Ayson et al. (1993) as an anaesthetic, has been shown to strongly stimulate plasma prolactin levels in tilapia (B. Auperin \& P. Prunet, unpublished observations). Thus, these data cannot really be compared with our results obtained under non-stressful conditions.

The amounts of tiPRL $L_{1}$ and tiPRL ${ }_{I I}$ in the pituitaries also exhibited different patterns of change after transfer. A drop in tiPRL $L_{I}$ content was observed between the first half-day and the third day after transfer, with a sharper drop by day 7 and thereafter. By day 14, the tiPRL $L_{I}$ content was only one-twelfth of its initial value. The tiPRL ${ }_{I I}$ content, on the other hand, had decreased only by half after 2 weeks in brackish water. The fact that tiPRL ${ }_{I I}$, unlike tiPRL $\mathrm{I}_{\mathrm{I}}$, is present at significant levels in fish adapted to brackish water suggests that the two prolactins have different osmoregulatory roles during adaptation to a hyperosmotic environment.

To test this hypothesis further, we investigated the effects of repeated injections of recombinant tiPRL $_{I}$ or tiPRL $L_{I I}$ in fish adapted to brackish water. Interestingly, tiPRL $\mathrm{I}_{\mathrm{I}}$ behaved like ovine prolactin in that it induced a dose-dependent ion-retaining effect, as shown by the increased $\mathrm{Na}^{+}$and $\mathrm{Cl}^{-}$ levels. This is in keeping with results obtained with ovine prolactin or tilapia pituitary extracts (Clarke, 1973a,b; Dharmamba \& Maetz, 1976). In the present study, tiPRL $\mathrm{I}_{\mathrm{I}}$ was between 50 and 100 times more potent than ovine prolactin. The increase caused by tiPRL ${ }_{I I}$, on the other hand, was markedly smaller and not dose-related. Since the two tiPRL forms produced in $E$. coli exhibit similar potencies in two different bioassays and are as biologically active as natural tiPRLs (Swennen et al. 1991; Auperin et al. 1993), the results obtained in this particular test cannot be attributed to differences in the ability of the two recombinant prolactins to perform their biological functions. Moreover, as suggested by Swennen et al. (1991), Auperin et al. (1993) have recently demonstrated the presence of only one class of tiPRL receptor in gill and kidney, which binds tiPRL $L_{I}$ with a higher affinity than tiPRL $L_{I I}$. This allowed us to exclude the hypothesis that the low endogenous plasma levels of tiPRL $_{I I}$ would have interfered (at the level of receptor) with exogenous tiPRL treatment; in such a case, a similar effect would have been observed with both tiPRL forms. We conclude that the significant level of tiPRL ${ }_{I I}$ measured in fish adapted to a hyperosmotic environment can be linked with the fact that this hormone exerts a limited ion-retaining effect. This further tallies with the observation that tiPRL $L_{I}$, which affects the hydromineral balance much more drastically, is undetectable under the same conditions. We conclude from this that the two prolactins have different osmoregulatory roles in $O$. niloticus adapted to brackish water.

Previous studies comparing the osmoregulatory effects of the two tiPRLs used biological assays based on the physiology of fresh water osmoregulation, including the $\mathrm{Na}^{+}$-retaining effect in hypophysectomized tilapia $(O$. mossambicus) reared in fresh water (Specker et al. 1985; Young et al. 1988), effects on transepithelial potential measurements in hypophysectomized tilapia (Young et al. 1988), and effects on plasma $\mathrm{Ca}^{2+}$ levels (Swennen et al. 1991). In all cases, both forms exhibited similar biological activities and their osmoregulatory effects resembled those described for ovine prolactin (Dharmamba, 1970; Wendelaar Bonga \& Meis, 1981; Flik et al. 1984). A comparison with our results suggests that prolactin may exert different effects in hyperosmotic and hypo-osmotic environments, and that some of these effects may be mediated by one form only.

In tilapia adapted to sea water, ovine prolactin inhibits chloride secretion by the gills (Foskett et al. 1982) and reduces branchial efflux (Dharmamba \& Maetz, 1976), which results in an increased $\mathrm{Na}^{+}$level. Accordingly, Pisam et al. 
(1993) have recently shown that tiPRL ${ }_{I}$ significantly alters the ultrastructural features of gill chloride cells shifted from a 'sea water' to a 'fresh water' type. Together with the present study, these data indicate that tiPRL $\mathrm{I}_{\mathrm{I}}$ modifies gill chloride cell differentiation and the activity of these cells in a hyperosmotic environment. A similar effect was also observed after ovine prolactin injection (M. Pisam, unpublished observation). On the other hand, our present data on tiPRL effect of this form on chloride cell morphology (M. Pisam, unpublished observation) suggest that tiPRL $_{I I}$ might not be involved in the regulation of chloride cells. Foskett et al. (1983) have suggested that prolactin may not act on chloride cells in fresh water-adapted tilapia; the $\mathrm{Na}^{+}$-retaining effect of prolactin traditionally described in fresh waterreared specimens could be due to a decreased osmotic permeability to water in osmoregulatory organs (Dharmamba \& Maetz, 1972; Ogawa et al. 1973; Wendelaar Bonga \& Van Der Meij, 1980). According to Specker et al. (1985), both forms of tiPRL prevent the loss of $\mathrm{Na}^{+}$from the plasma of hypophysectomized tilapia kept in fresh water. In conclusion, we suggest that tiPRL $\mathrm{II}_{\mathrm{II}}$, in contrast to tiPRL $L_{I}$, does not affect chloride cells in a hyperosmotic environment, but does inhibit permeability to water, which could explain the slight but significant ion-retaining effect observed.

Upon transfer to brackish water, the ratio of tiPRL ${ }_{I I}$ to tiPRL $L_{I}$ was found to increase markedly in the pituitaries and plasma of transferred fish. This points to a differential regulation of the two forms in response to salinity changes, as regards their synthesis in the pituitary and subsequent release. This is in keeping with electrophoretic studies of pituitary tiPRL $\mathrm{I}_{\mathrm{I}}$ and tiPRL $\mathrm{L}_{\mathrm{II}}$ contents in fresh water- and sea water-adapted $O$. mossambicus (Borski et al. 1992). However, no differences were detected in the neuroendocrine control of synthesis or release of the two tiPRLs. Likewise, both tiPRL forms responded similarly when the actions of osmotic pressure and neuropeptides were tested on pituitary fragments (Rivas et al. 1986; Kelley et al. 1988). Clearly, it is necessary to conduct further studies on tilapia adapted to a hyperosmotic environment in order to clarify which endocrine factors mediate this differential regulation.

Further insight into the differential regulation of tiPRL synthesis was provided by our analysis of prolactin mRNA in $O$. niloticus adapted to fresh water and brackish water. Although both tiPRL cDNA probes hybridized with both mRNAs, differences in size and intensity enabled us to conclude that tiPRL ${ }_{I}$ and tiPRL $\mathrm{II}_{\mathrm{I}}$ are the respective translation products of the large $(1.7 \mathrm{~kb})$ and the small $(1.3 \mathrm{~kb})$ mRNAs. Environmental salinity modified the pituitary content of both tiPRL mRNAs as indicated by Northern blot analysis. This is in keeping with semi-quantitative in situ hybridization analysis of tiPRL mRNA in $O$. mossambicus transferred to sea water (Nishioka et al. 1993). Interestingly, after a 2 -week exposure to brackish water in the present study, both mRNA levels were found to have decreased similarly, despite the fact that the tiPRL $\mathrm{II}_{\text {II }}$ content was five times higher than the tiPRL ${ }_{I}$ content in the pituitaries of these fish (Fig. 3). This suggests that the differential regulation of the two forms occurs primarily at one or more post-transcriptional levels. Post-transcriptional regulation of prolactin synthesis has been described in the literature. In $\mathrm{GH}_{3}$ cells, treatment with $\mathrm{Ca}^{2+}$ significantly increases the level of prolactin mRNA by regulating posttranscriptional steps occuring very soon after the completion of transcription (Preston et al. 1990; Delidow et al. 1992). This does not affect the half-life of prolactin $\mathrm{mRNA}$ in the cytoplasm. In contrast, Gellersen et al. (1992) have observed an increase in steady-state levels of human prolactin in the IM-9-P3 cell line due to mRNA stabilization after retinoic acid treatment.

In conclusion, our evidence suggests that the two tiPRLs perform different osmoregulatory functions in $O$. niloticus adapted to brackish water. It further points to differential regulation of tiPRL $L_{I}$ and tiPRL $L_{I I}$ synthesis, probably at a post-transcriptional level, after transfer to a hyperosmotic environment.

\section{ACKNOWLEDGEMENTS}

The authors gratefully thank M. Gerber for excellent fish husbandry, G. Paboeuf for help in collecting the samples and Y. Le Graët (Laboratoire de Technologie Laitière, INRA) for use of the atomic absorption spectrophotometer. We also thank IDESSA (Institut des Savanes, Département des Productions Animales, Bouaké, Ivory Coast) for providing the tilapia. This work was supported in part by a grant from CIRAD.

\section{REFERENCES}

Auperin, B., Rentier-Delrue, F., Martial, J. \& Prunet, P. (1993). Prolactin receptors in osmoregulatory organs of tilapia (Oreochromis niloticus). I.U.B.S. Symposium: Advances in the Molecular Endocrinology of Fish, Toronto, Canada, 23-25 May 1993, S10 (Abstract).

Ayson, F. G., Kaneko, T., Tagawa, M., Hasegawa, S., Grau, E. G., Nishioka, R. S., King, D. S., Bern, H. A. \& Hirano, 
T. (1993). Effects of acclimation to hypertonic environment on plasma and pituitary levels of two prolactins and growth hormone in two species of tilapia, Oreochromis mossambicus and Oreochromis niloticus. General and Comparative Endocrinology 89, 138-148.

Borski, R. J., Hansen, M. U., Nishioka, R. S. \& Grau, E. G. (1992). Differential processing of two prolactins of the tilapia (Oreochromis mossambicus) in relation to environmental salinity. Fournal of Experimental Zoology 264, 46-54.

Brooks, C. L., Kim, B. G., Aphale, P., Kleeman, B. E. \& Johnson, G. C. (1990). Phosphorylated variant of bovine prolactin. Molecular and Cellular Endocrinology 71, 117-123.

Chomczynski, P. \& Sacchi, N. (1987). Single-step method of RNA isolation by acid guanidium thiocyanate-phenolchloroform extraction. Analytical Biochemistry 161, $156-159$

Clapp, C., Sears, P. S., Russel, D. H., Richards, J., Levay-Young, B. K. \& Nicoll, C. S. (1989). Biological and immunological characterization of cleaved $16 \mathrm{~K}$ forms of rat prolactin. Endocrinology 122, 2892-2898.

Clarke, W. C. (1973a). Sodium retaining bioassay of prolactin in the intact teleost Tilapia mossambica acclimated to sea water. General and Comparative Endocrinology 21, 498-512.

Clarke, W. C. (1973b). Disc-electrophoretic identification of prolactin in the cichlid teleosts Tilapia and Cichlasoma and densitometric measurement of its concentration in Tilapia pituitaries during salinity transfer experiments. Canadian Fournal of Zoology 51, 687-695.

Clarke, W. C. \& Bern, H. A. (1980). Comparative endocrinology of prolactin. In Hormonal Proteins and Peptides, vol. 8, pp. 105-197. Ed. C. H. Li. New York: Academic Press.

Delidow, B. C., Lail-Trecker, M. \& White, B. A. (1992). Effects of calcium and calcium ionophores on prolactin gene expression in $\mathrm{GH}_{3}$ and 235-1 rat pituitary tumor cells. Molecular Endocrinology 6, 1268-1276.

Dharmamba, M. (1970). Studies of the effects of hypophysectomy and prolactin on plasma osmolarity and plasma sodium in Tilapia mossambica. General and Comparative Endocrinology' 14, 256-269.

Dharmamba, M., Bornancin, M. \& Maetz, J. (1975). Environmental salinity and sodium and chloride exchanges across the gill of Tilapia mossambica. Fournal de Physiologie 70, 627-636.

Dharmamba, M., Handin, R. I., Nandin, J. \& Bern, H. A. (1967). Effect of prolactin on freshwater survival and on plasma osmotic pressure of hypophysectomized Tilapia mossambica. General and Comparative Endocrinology 9, 295-302.

Dharmamba, M. \& Maetz, J. (1972). Effects of hypophysectomy and prolactin on the sodium balance in Tilapia mossambica in freshwater. General and Comparative Endocrinology 19, 175-183.

Dharmamba, M. \& Maetz, J. (1976). Branchial sodium exchange in seawater-adapted Tilapia mossambica: effects of prolactin and hypophysectomy. Fournal of Endocrinology 70, 293-299.

Flik, G., Wendelaar Bonga, S. E. \& Fenwick, J. C. (1984). $\mathrm{Ca}^{2+}$-dependent phosphatase and $\mathrm{Ca}^{2+}$-dependent ATPase activities in plasma membranes of eel epithelium - III. Stimulation of branchial high-affinity $\mathrm{Ca}^{2+}$-ATPase activity during prolactin-induced hypercalcemia in American eels. Comparative Biochemistry and Physiology 79B, 521-524.

Foskett, J. K., Bern, H. A., Machen, T. E. \& Conner, M. (1983). Chloride cells and the hormonal control of teleost fish osmoregulation. Fournal of Experimental Biology 106, $255-281$
Foskett, J. K., Machen, T. E. \& Bern, H. A. (1982). Chloride secretion and conductance of teleost opercular membrane: effects of prolactin. American Fournal of Physiology 242, R380-R389.

Gellersen, B., Kempf, R., Hartung, S., Bonhoff, A. \& DiMattia, G. E. (1992). Posttranscriptional regulation of the human prolactin gene in IM-9-P3 cells by retinoic acid. Endocrinology 131, 1017-1025.

Greenwood, F. C., Hunter, W. M. \& Glover, J. S. (1963). The preparation of ${ }^{131}$ I labelled human $\mathrm{GH}$ of high specific radioactivity. Biochemical fournal 89, 114-123.

Hirano, T. (1986). The spectrum of prolactin action in teleosts. In Comparative Endocrinology: Developments and Directions, pp. 53-74. Ed. C. L. Ralph. New York: Alan R. Liss Inc.

Hirano, T., Prunet, P., Kawauchi, H., Takahashi, A., Ogasawara, T., Kubota, J., Nishioka, R. S., Bern, H. A., Takada, K. \& Ishii, S. (1985). Development and validation of a salmon prolactin radioimmunoassay. General and Comparative Endocrinology 59, 266-276.

Kelley, K. M., Nihioka, R. S. \& Bern, H. A. (1988). Novel effect of vasoactive intestinal polypeptide and peptide histidine isoleucine: inhibition of in vitro secretion of prolactin in the tilapia Oreochromis mossambicus. General and Comparative Endocrinology 72, 97-106.

Le Bail, P. Y., Sumpter, J. P., Carragher, J. F., Mourot, B., Niu, P. D. \& Weil, C. (1991). Development and validation of a highly sensitive radioimmunoassay for chinook salmon (Oncorhynchus tshawytscha) growth hormone. General and Comparative Endocrinology 83, 75-85.

Lewis, U. J., Singh, R. N. P., Lewis, U. J., Seavey, B. K. \& Sinha, Y. N. (1984). Glycosylated ovine prolactin. Proceedings of the National Academy of Sciences of the U.S.A. 81, 385-389.

Loretz, C. A. \& Bern, H. A. (1982). Prolactin and osmoregulation in vertebrates. Neuroendocrinology 35, 292-304.

Martal, J. (1972). Détermination radioimmunologique des hormones de croissance ovine et bovine: étude des conditions d'iodation et obtention d'une hormone somatotrope radioiodée sans prolactine contaminante. Comptes Rendus de l'Académie des Sciences 274, 2893-2896.

Nicoll, C. S., Wilson, S. W., Nishioka, R. S. \& Bern, H. A. (1981). Blood and pituitary prolactin levels in tilapia (Sarotherodon mossambicus; Teleostei) from different salinities as measured by a homologous radioimmunoassav. General and Comparative Endocrinology 44, 365-373.

Nishioka, R. S. (1980). Hypophysectomy of tilapia Sarotherodon mossambicus through the orbit. General and Comparative Endocrinology 40, 377-378.

Nishioka, R. S., De Jesus, E. G. T. \& Hyodo, S. (1993). Localization of mRNAs for a pair of prolactins and growth hormone in the tilapia pituitary using in situ hybridization with oligonucleotide probes. General and Comparative Endocrinology 89, 72-81.

Ogawa, M., Yagasaki, M. \& Yamazaki, F. (1973). The effect of prolactin on water influx in isolated gills of the goldfish, Carassius auratus L. Comparative Biochemistry and Physiology 44A, 1951-1955.

Pakdel, F., Le Guellec, C., Vaillant, C., Le Roux, M. G. \& Valotaire, Y. (1989). Identification and estrogen induction of two estrogen receptor (ER) messenger ribonucleic acids in the rainbow trout liver: sequence homology with other ERs. Molecular Endocrinology 3, 44-51.

Pisam, M., Aupérin, B., Prunet, P., Rentier-Delrue, F., Martial, J. \& Rambourg, A. (1993). Effects of prolactin on $\alpha$ and $\beta$ chloride cells in the gill epithelium of the saltwater adapted tilapia Oreochromis niloticus. Anatomical Record 235, $275-284$. 
Preston, G. M., Billis, W. M. \& White, B. A. (1990).

Transcriptional and posttranscriptional regulation of the rat prolactin gene by calcium. Molecular and Cellular Biology 10, $442-448$.

Prunet, P., Avella, M., Fostier, A., Björnsson, B. Th., Boeuf, G. \& Haux, C. (1990). Role of prolactin in salmonids. In Progress in Comparative Endocrinology, pp. 547-552. Eds A. Epple, C. G. Scanes \& M. T. Stetson. New York: Wiley-Liss.

Prunet, P., Boeuf, G. \& Houdebine, L. M. (1985). Plasma pituitary prolactin levels in rainbow trout during adaptation to different salinities. Fournal of Experimental Zoology 235, 187-196.

Prunet, P. \& Houdebine, L. M. (1984). Purification and biological characterization of chinook salmon prolactin. General and Comparative Endocrinology 53, 49-57.

Rentier-Delrue, F., Swennen, D., Prunet, P., Lion, M. \& Martial, J. A. (1989). Tilapia prolactin: molecular cloning of two cDNAs and expression in Echerichia coli. DNA 8, 261-270.

Rivas, R. J., Nishioka, R. S. \& Bern, H. A. (1986). In vitro effects of somatostatin and urotensin II on prolactin and growth hormone secretion in tilapia Oreochromis mossambicus. General and Comparative Endocrinology 63, 245-251.

Specker, J. L., Brown, P. S. \& Brown, S. C. (1989). Unequal activities of the two tilapia prolactins in the whole-animal transepithelial potential bioassay using the red eft. Fish Physiology and Biochemistry 7, 119-124.

Specker, J. L., King, D. S., Nishioka, R. S., Shirahata, K., Yamaguchi, K. \& Bern, H. A. (1985). Isolation and partial characterization of a pair of prolactins released in vitro by pituitary of a cichlid fish, Oreochromis mossambicus. Proceedings of the National Academy of Sciences of the U.S.A. 82, 7490-7494.

Suzuki, R. \& Hirano, T. (1991). Development of a homologous radioimmunoassay for eel prolactin. General and Comparative Endocrinology 81, 403-409.

Swennen, D., Rentier-Delrue, F., Auperin, B., Prunet, P., Flik, G., Wendelaar Bonga, S. E., Lion, M. \& Martial, J. A. (1991). Production and purification of biologically active recombinant tilapia (Oreochromis niloticus) prolactins. Fournal of Endocrinology 131, 219-227.
Thomas, P. S. (1980). Hybridization of denatured RNA and small DNA fragments transferred to nitrocellulose. Proceedings of the National Academy of Sciences of the U.S.A. 77, 5201-5205.

Wendelaar Bonga, S. E. \& Meis, S. (1981). Effects of external osmolarity, calcium and prolactin on growth and differentiation of the epidermal cells of the cichlid teleost Sarotherodon mossambicus. Cell and Tissue Research 221, 109-123.

Wendelaar Bonga, S. E. \& Van Der Meij, J. C. A. (1980). The effect of ambient calcium on prolactin cell activity and plasma electrolytes in Sarotherodon mossambicus (Tilapia mossambica). General and Comparative Endocrinology 40, 391-401.

Yamaguchi, K., Specker, J. L., King, D. S., Yokoo, Y., Nishioka, R. S., Hirano, T. \& Bern, H. A. (1988). Complete amino acid sequences of a pair of fish (Tilapia) prolactins, tPRL $_{177}$ and $\mathrm{PPL}_{188}$. Fournal of Biological Chemistry 263, 9113-9121.

Yasuda, A., Itoh, H. \& Kawauchi, H. (1986). Primary structure of chum salmon prolactins: occurrence of highly conserved regions. Archives of Biochemistry and Biophysics 244, $528-541$.

Yasuda, A., Miyazima, K., Kawauchi, H., Peter, R. E., Lin, H. R., Yamaguchi, K. \& Sano, H. (1987). Primary structure of common carp prolactins. General and Comparative Endocrinology 66, 280-290.

Young, P. S., McCormick, S. D., Demarest, J. R., Lin, R. J., Niskioka, R. S. \& Bern, H. A. (1988). Effects of salinity, hypophysectomy, and prolactin on whole-animal transepithelial potential in the tilapia Oreochromis mossambicus. General and Comparative Endocrinology 71, 389-397.

REVISED MANUSCRIPT RECEIVED 5 July 1993 